\title{
Speckle activity images based on the spatial variance of the phase
}

\author{
Héctor Rabal, Nelly Cap, Marcelo Trivi, Ricardo Arizaga, Alejandro Federico, \\ Gustavo E. Galizzi, and Guillermo H. Kaufmann
}

\begin{abstract}
We propose the display of the local spatial variance of the temporal variations of the phase as an activity descriptor in dynamic speckle images. The spatial autocorrelation of the speckle intensity is calculated in sliding windows, and an estimation of the variance of the phase variations in each region of the sample is determined. The activity images obtained in this way depict some interesting features and in some cases they could be related to physical magnitudes in the samples. A simulation is presented, and examples corresponding to usual study cases are also shown, namely, fruit bruising and paint drying. (C) 2006 Optical Society of America

OCIS codes: $110.0110,110.6150,030.6140$.
\end{abstract}

\section{Introduction}

Dynamic speckle or biospeckle is a phenomenon occurring when laser light illuminates scattering surfaces with some type of activity.1,2 For example, fruits, seeds, and painted surfaces subjected to drying show this behavior. The study of the temporal evolution of the speckle patterns may provide an interesting tool to characterize the parameters involved in some biological applications and industrial processes.

The autocorrelation analysis of the intensity as a function of time is a classical method for the characterization of dynamic speckle patterns. ${ }^{3,4}$ FWHM is a possible estimator of speckle lifetime and consequently of the local activity. Another approach to analyze the dynamic speckle is given by the generation of the time history of the speckle pattern (THSP) and its characterization using different descriptors. Several algorithms were proposed to give a quantitative measurement of the speckle activity using the

H. Rabal (hrabal@ing.unlp.edu.ar), N. Cap, M. Trivi, and R. Arizaga are with the Centro de Investigaciones Opticas and UID Optimo, Facultad de Ingeniería, Universidad National de la Plata, P. O. Box 124, 1900 La Plata, Argentina. A. Federico is with the Física y Metrología, Instituto Nacional de Tecnología Industrial, P. O. Box B1650WAB, B1650KNA San Martín, Argentina. G. E. Galizzi and G. H. Kaufmann are with the Instituto de Física Rosario (CONICET-UNR), Boulevard 27 de Febrero 210 bis, S2000EZP Rosario, Argentina.

Received 9 December 2005; revised 4 April 2006; accepted 9 May 2006; posted 19 July 2006 (Doc. ID 66474).

$0003-6935 / 06 / 348733-06 \$ 15.00 / 0$

(C) 2006 Optical Society of America
THSP.5,6 Although several methods are effective in some cases, most of them are difficult to associate with physical changes that occur in the sample.

In the activity images corresponding to dynamic speckle, the sizes, shapes, and spatial distribution of the areas of the same gray levels change with time. There are several methods to generate a qualitative display with a speckle pattern activity. ${ }^{2,7-9}$ In these cases, a gray level or false color display shows different regions of the samples that allow us to discriminate the differential activity of the scattering surfaces.

Some of the display methods already developed consist of heuristic tools that give little insight into the physical origins that can be attributed to the observed activity. As this estimation is an inverse problem, the causes of the activity most probably cannot be uniquely determined unless some $a$ priori knowledge of the phenomenon is available. Nevertheless, under some not very stringent assumptions, estimations of the spatial variance of the phase between consecutive frames can be evaluated, and based on these results, segmentation image displays can also be obtained.

Phase can be easily associated with its physical origins. As a matter of fact, the origin of dynamic speckle phenomena is mainly due to random phase differences, Doppler effect, beating of waves with close frequencies, etc. If the reflectance of an object is constant, temporal variations of the intensity in its speckle patterns are due to phase changes. Therefore if the physical origin of the phase change is known or suspected, the processing leads to additional infor- 
mation on the underlying phenomena occurring in the sample.

Recently, we presented a numerical simulation that allows for the generation of temporal sequences of $2 \mathrm{D}$ dynamic speckle patterns. ${ }^{10}$ In this paper we propose an estimation of the spatial variance of the phase from the intensity autocorrelation as a descriptor of the local activity of dynamic speckle images. In the next section, we will briefly describe the theory of the intensity variations in a speckle pattern generated by moving scatterers. Then we will show how an estimation of the spatial variance of phase can be calculated from its intensity autocorrelation. Finally, numerical simulations of a typical experiment will be shown, and the results obtained from two actual experiments on study cases will be presented, namely, the drying of paint on a coin and the presence of bruising in a fruit. In these cases, the knowledge of the height differences in the profile of a coin and the presence of induced bruising in an apple are examples of a priori available knowledge that helps to interpret the results.

\section{Theory}

In this work we have used the same formulation already proposed in Ref. 10 as briefly described below. In this simplified model, the surface of the sample under study is considered to be composed of a set of discrete scattering centers. When coherent polarized laser light with wavelength $\lambda$ is used for the illumination, the amplitude of the electric field $U_{0}$, at a point in the Fresnel region, can be calculated as

$$
U_{0}=\sum_{j} a_{j} \exp \left(\mathrm{i} \phi_{j}\right)
$$

where $a_{\bar{j}}$ and $\phi_{j}$ are the amplitude and phase contributions due to the scattering center $j$. The usual assumptions that lead to the definition of a welldeveloped speckle pattern are also assumed here.

When the sample shows local activity, the amplitude of the electric field can be written as

$$
U=\sum_{j} a_{j} \exp \left\lfloor i\left(\phi_{j}+\Delta \phi_{j}\right)\right\rfloor
$$

where $\Delta \phi_{j}$ is the change in the optical phase because of the activity.

Assuming that a set of $K$ speckle patterns is sequentially acquired at equal time steps, a quantitative measurement of the activity of the underlying process can be obtained through the evaluation of the spatial correlation coefficient $c(k)$ between the first image and the following ones. Considering that the process is piecelike stationary during the acquisition time, $c(k)$ is defined by

$$
c(k)=\frac{\langle I(0) I(k)\rangle-\langle I(0)\rangle\langle I(k)\rangle}{\left\{\left[\left\langle I^{2}(0)\right\rangle-\langle I(0)\rangle^{2}\right]\left[\left\langle I^{2}(k)\right\rangle-\langle I(k)\rangle^{2}\right]\right\}^{1 / 2}},
$$

where $I(k)$ is the intensity at a point of each temporal frame $k$ and \langle\rangle denotes the spatial mean value operator over each speckle image of the sequence.

When the Doppler effect is the dominant cause of the activity, the phase change $\Delta \phi_{j}$ may be due to an alteration $\Delta h_{j}$ in the height of the scattering centers normal to the surface. Then,

$$
\Delta \phi_{j}=(2 \pi / \lambda)\left(1+\cos \theta_{j}\right) \Delta h_{j}
$$

where $\theta_{j}$ is the angle between the illuminating beam and the observation direction normal to the surface at the scattering center $j$.

It was demonstrated in Refs. 11 and 12 that the correlation coefficient $c(k)$ decreases exponentially with the variance of the phase change. This result was obtained under the assumption that all amplitude and phase changes of the scattering centers are statistically independent of each other and of the amplitudes and phases of all other scatterers, that the phase change $\Delta \phi_{j}$ given by Eq. (4) follows a normal distribution with a variance $\sigma^{2}(\Delta \phi)$, and that there are no changes in the reflectivity of the sample. Consequently, the correlation coefficient can be expressed as

$$
c(k)=\exp \left[-\sigma^{2}(\Delta \phi)\right]
$$

where the phase change $\Delta \phi$ must be evaluated with respect to the first speckle pattern of the temporal sequence $(k=0)$. Equation (5) holds both for objective and for subjective speckle patterns, as long as the surface roughness and the microstructure variation cannot be resolved. Therefore local estimations of the phase variance can be calculated if the regions where the space average is performed are large enough for the statistics to be effective.

\section{Computer Simulation of Time Varying} Speckle Patterns

Sequences of time varying speckle patterns can be computer simulated using the method described in Ref. 10 . The intensity $I(m, n, k)$ in each speckle pattern point belonging to a given temporal sequence is evaluated from

$$
I(m, n, k)=\left|F^{-1}\left\{H F\left[e^{\mathrm{i} \phi(m, n, k)}\right]\right\}\right|^{2},
$$

where $m=n=0,1, \ldots, N-1$ are the pixel coordinates of the CCD detector used to acquire the speckle images, $\phi(m, n, k)$ is an $N \times N$ matrix, which represents the phase distribution of the scattered light from the rough surface; $i$ is the imaginary unit; $F$ and $F^{-1}$ are the direct and inverse Fourier transforms, respectively; and $H$ is a circular low-pass filter with radius $r$ in the Fourier space, which represents the circular aperture of the camera lens and determines the average speckle size of the speckle grains.

The speckle phase $\phi(m, n, 0)$ corresponding to the first frame $k=0$ is chosen as a matrix with uniform distributed values in the interval $(-\pi, \pi)$. Considering that all scattering centers move randomly up and 
down along the direction normal to the surface of the sample, the phase $\phi(m, n, k)$ is evaluated from

$$
\begin{aligned}
\phi(m, n, k)= & \phi(m, n, k-1)+G(m, n, k) \\
& \times \sigma[\Delta \phi(m, n, k-1, k)], \\
& k=1, \ldots, K-1,
\end{aligned}
$$

where $\sigma[\Delta \phi(m, n, k-1, k)]$ is the standard deviation of the phase change between two consecutive speckle patterns, and $G(m, n, k)$ is an $N \times N$ random matrix with Gaussian distributed values having a mean $\mu=0$, and a standard deviation $\sigma=1$, which has to be recalculated at every frame $k$.

Through a simple mathematical derivation, the standard deviation of the phase change between two consecutive speckle patterns $\sigma[\Delta \phi(m, n, k-1, k)]$ can be expressed in terms of the correlation coefficient $c(k)$ of each image with respect to the first frame of the sequence as

$$
-\ln [c(k)]=-\ln [c(k-1)]+\sigma[\Delta \phi(k-1, k)]^{2} .
$$

Therefore the sequence of computer simulated speckle patterns generated by Eq. (6) is produced using a phase distribution given by

$$
\begin{aligned}
\phi(m, n, k)= & \phi(m, n, k-1)+G(m, n, k) \\
& \times \sqrt{\ln c(k-1)-\ln c(k)} .
\end{aligned}
$$

The simulation model requires $c(k)$ to be a monotonically decreasing function. This is a limitation of the model, but this is the case that appears at least in several processes such as paint drying and metal corrosion. If this requirement is not verified or due to noise, the term inside the square root of Eq. (9) could be negative in some pixels or regions, and then $\sigma$ in Eq. (8) will not represent the phase variance. Even when the expression inside the square root of Eq. (9) is not negative, this is not a guarantee that the conditions required for the model are fulfilled. However, we are going to see that even in these cases, the processed image gives some useful qualitative information on the distribution of the activity in the object.

\section{Experiments and Results}

We used the described numerical model to generate speckle activity images based on the spatial variance of the phase. To test for the possibility of obtaining segmentation images based on the variance of phase changes, we numerically simulated a sequence of active speckle images with different values of the variance in two different regions. Afterward, the simulated speckle patterns were processed as follows: For each pixel of the speckle patterns, the $c(1)$ value was calculated from the first two frames 0 and 1 by means of Eq. (3) using a $5 \times 5$ window. The window size is given by a trial and error optimization com-

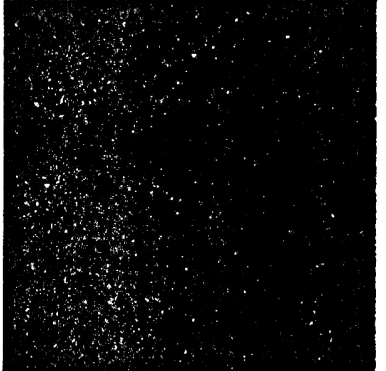

(a)

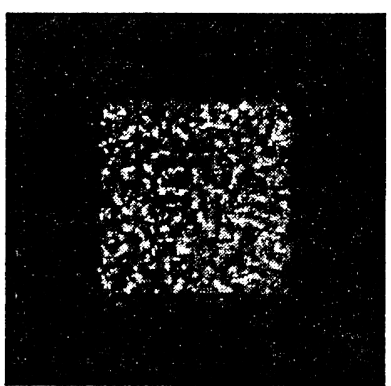

(b)
Fig. 1. (a) Typical speckle pattern produced by numerical simulation; (b) display of the processed image where the loci of equal $\sigma$ values are clearly perceived.

promise between statistics and resolution. Large windows reduce spatial resolution and improve variance precision, while the converse is true for small windows.

The same calculation was performed with the frames 0 and 2 to obtain $c(2)$. A map of $c(1)$ and $c(2)$ was obtained by displacing the window over the speckle patterns. Using these maps, an estimation of the local value of $\sigma$ was calculated by means of Eq. (8). Then this procedure was repeated for all the consecutive sets of three frames belonging to a given temporal sequence. Finally, the temporal average of the previously computed $\sigma$ values was evaluated.

The space averaging operation that is applied inside the window and is required in the calculation of the variance is in conflict with high space resolution in the resulting image. The use of a sliding window results in a tiling appearance of the image. To reduce this deleterious effect, we decided to perform the estimation over a set of consecutive frames taken in groups of three and to average the results.

Figure 1(a) shows one of these simulated speckle patterns where the spatial phase variations were set to different values in two square regions. Although no hint of that variation can be observed in the previous image, Fig. 1(b) displays the processed image in which the loci of equal $\sigma$ values are clearly perceived.

Afterward, we performed several experiments with actual speckle patterns already used in previous works. The temporal sequences of speckle patterns were acquired using the setup shown in Fig. 2. A divergent laser beam ( $\lambda=632.8 \mathrm{~nm}$, polarized) was used to illuminate the object while a CCD camera acquired the dynamic speckle patterns and a frame grabber digitized and stored the intensity data for each frame. The experimental conditions were: pixel size, $8.4 \mu \mathrm{m} \times 9.8 \mu \mathrm{m}$; magnification, $1 / 3$; focal distance of the objective, $50 \mathrm{~cm}$ (with added close-up rings); pupil size, $3.1 \mathrm{~mm}$; illumination angle, $\sim 45^{\circ}$. A region wider than the object was illuminated to assure that the object has received uniform illumination.

Then, the spatial correlation evaluated inside the sliding windows was calculated for sets of three consecutive frames as described above. The variance of 


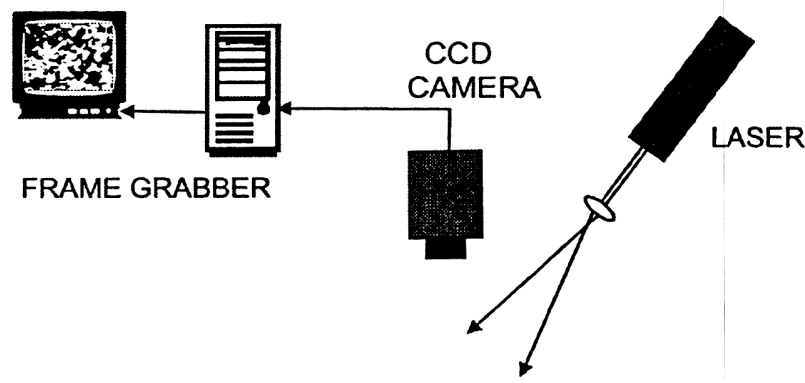

SAMPLE

Fig. 2. Experimental setup used to acquire speckle pattern sequences.

the phase was estimated using Eq. (8), and the activity image was generated using scaled values of the calculated variance. For the assumptions stated in the theory to be valid and the phase variance to be accurately determined, the speckle pattern must be well sampled. This means that the mean speckle size should be greater than the pixel size. This fact was also taken into account both in the numerical simulations and in the experiments. The average speckle size used both in the computer simulation and in the experiments was 2 pixels. Noise in the CCD camera, in the laser source, and in the image processor, limits the performance of the method. Because of the noise, activity changes meaning differences in $\sigma$ smaller than $0.1 \mathrm{rad}$ cannot be accurately detected.

As explained in Ref. 10, paint drying is a process that was found to be well fitted to apply the described theoretical model. For this reason, one test involved the painting of a coin with a layer of spatial variable thickness due to its relief. It was expected that the paint located in thinner layers, because of the protruding parts of the coin, would dry faster and therefore would be less active than in thicker regions. Figure 3(a) shows the original coin illuminated by incoherent light. One frame of the sequence of speckle patterns is depicted in Fig. 3(b) where no hint of the coin relief can be perceived. Figure 3(c) shows the processed image obtained using the proposed method. Thinner painted regions appear darker than the thicker ones, thus indicating that they are drier and consequently less active and display smaller variance of the phase variations. Note that not only coarse features, such as the numbers on the coin, but also finer detail as on the rim, can also be observed.

We have also performed a test on a controlled bruising experiment on an apple. This is an object that had previously been used as a case study to evaluate other display techniques.9,13=15 The object was a region of an apple where intentional bruising was produced by letting a calibrated ball with a diameter of $21.9 \mathrm{~mm}$ and a weight of $133.6 \mathrm{~g}$ fall from a height of $20 \mathrm{~cm}$. The bruising could not be visually perceived. An inert object (a piece of metal) was in-

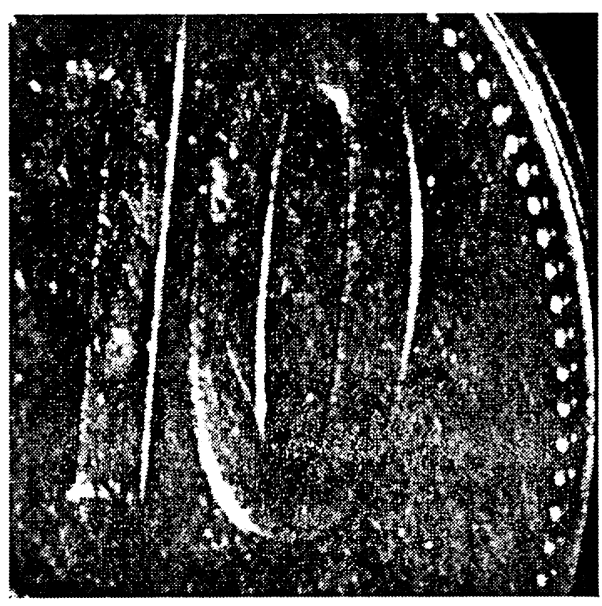

(a)

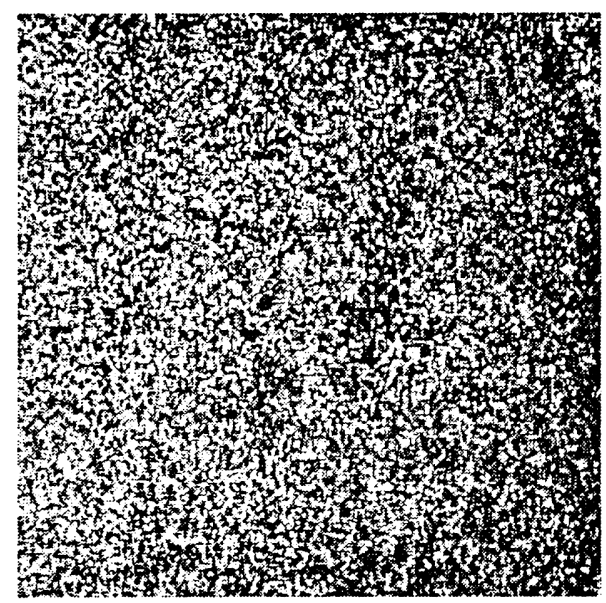

(b)

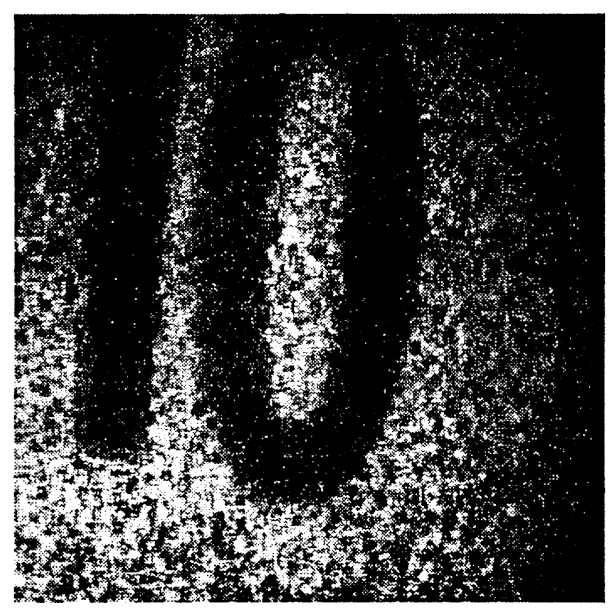

(c)

Fig. 3. (a) Coin observed under incoherent light illumination; (b) speckle pattern of the coin illuminated by laser light (the imaged area is $-15 \mathrm{~mm} \times 15 \mathrm{~mm}$ ); (c) display of the processed image where the loci of equal activity corresponding to equal thickness of paint appear as gray levels. 


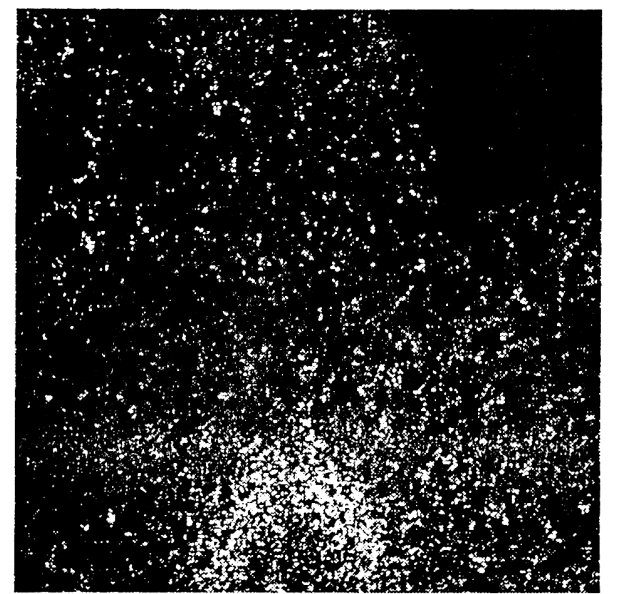

Fig. 4. Display of the activity in a bruised apple experiment (the imaged area is $220 \mu \mathrm{m} \times 20 \mathrm{~mm}$ ). The upper right corner shows an inert object included as a reference, and it appears dark. The bruised region is in the middle to lower region of the image and appears with a bright rim indicating higher activity than in the healthy regions.

cluded as a reference surface in the upper right corner of the image.

As this phenomenon includes biological tissue, its inner dynamics are poorly known, and it is not to be expected that the conditions of validity of the proposed model be fulfilled. We found that many errors occurred as manifested by obtaining negative values of $\sigma^{2}$. In this case, one out of three pixels showed this behavior. We constructed an image excluding these results, and nevertheless the three regions can be clearly observed as seen in Fig. 4. The inert region appears dark, while the healthy regions of the apple, with intermediate activity, appear in an intermediate gray level. The bruised region located in the middle to bottom of the image appears very bright, indicating higher activity.

The images obtained by applying this methodology are not high-resolution ones, as also happens with some other methods involving spatial windows as, for example, with laser speckle contrast analysis. ${ }^{2}$ Speckle activity images show some pixeling and also edge smoothing. Therefore it is expected that edges will not be well resolved. Sharp activity edges are not commonplace in biological tissues so that in most cases this does not constitute a severe limitation. Nevertheless, notice that on the edge that appears on the border between the inert material and the apple in the upper right corner of Fig. 4, the method distinguishes the activity of both regions quite well.

\section{Conclusions}

In each of the developed methods, to estimate dynamic speckle activity, a different magnitude is calculated and so are the resulting images. Some activity images are sensitive to the temporal signal roughness (Hurst coefficient ${ }^{14}$ ), some others to entropy, ${ }^{15}$ others to the proportion of moving scattering centers, ${ }^{16}$ and others to the energy present in different temporal frequency bands, ${ }^{9}$ to name just a few. In all cases, the $a$ priori knowledge of the phenomenon under study can help in the choice of the method to be used, and an expert in the phenomenon under study helps to discern the best choice.

In the method that we are proposing here, the observed result reflects the spatial variance of the phase if the required conditions are fulfilled. That magnitude can be associated with the velocity of the scattering centers or to refractive index variations or to other physical features of the surface that could be $a$ priori known. It gives different information that had not been previously exploited in this context. In some simplified nonbiological examples such as corrosion, ${ }^{12}$ the variance of phase can be interpreted as due to the temporal changes in the height of the surface; a well-defined physical property, but biological processes are not so simple and some ambiguity should always be expected.

The phase variance determined from the spatial autocorrelation of the intensity of consecutive frames in a sequence of dynamic speckle patterns can be used as an estimator of its activity, and if some additional information on its origin is available, it can be related to its physical origin. Activity images obtained with the proposed procedure then add to the understanding and segmentation of active regions and can be used for screening of known phenomena when they satisfy the adequate statistics. A compromise between statistical significance of the estimation and spatial resolution in the resulting image display is implicit. However, this effect can be partially alleviated by averaging several sets of consecutive results when the phenomenon is stationary.

Although in many cases the Doppler shift is the main origin of the dynamics, this is not always the case. Refractive index time variations, multiple scattering, and shading are other possible causes, and these effects were not taken into account in the simulations nor were they adequately interpreted in the experimental results, if they were present. Even if the statistical assumptions are satisfied, the variance of the phase still can be attributed to more than one cause.

It should be noted that the computation of $\sigma^{2}(\Delta \phi)$ involves the ratio of two values of the normalized correlation. In poorly illuminated areas, noise can possibly be boosted, and erroneous values of the variance will be obtained. Therefore care should be taken to maintain a uniform illumination over the whole sample.

The need for using sliding windows limits both spatial and intensity resolutions. Variations in reflectivity were assumed not to be present. Nevertheless, dynamic scattering is known to be a process of many to one; that is, similar effects can be due to different causes. Therefore the present work should be considered as a step forward to obtain more information on the spatial variation of active samples from their speckle patterns. 
This research has been supported by Agencia Nacional de Promoción Científica y Tecnológica, Consejo Nacional de Investigaciones Científicas y Técnicas, Comisión de Investigaciones Científicas de la Provincia de Buenos Aires, Red Argentina de Óptica and Universidad Nacional de La Plata, all from Argentina.

\section{References}

1. Y. Aizu and T. Asakura, "Biospeckle," in Trends in Optics, A. Consortini, ed. (Academic, 1996), Chap. 2.

2. J. D. Briers and S. Webster, "Laser speckle contrast analysis (LASCA): a nonscanning, full field technique for monitoring capillary blood flow," J. Biomed. Opt. 1, 174-179 (1996).

3. Y. Aizu and T. Asakura, "Biospeckle phenomena and their applications to the evaluation of blood flow," Opt. Laser Technol. 23, 205-219 (1991).

4. B. Ruth, "Superposition of two dynamic speckle patterns. An application to noncontact blood flow measurements," J. Mod. Opt. 34, 257-273 (1987).

5. A. Oulamara, G. Tribillon, and J. Dovernoy, "Biological activity measurements on botanical specimen surfaces using a temporal decorrelation effect of laser speckle," J. Mod. Opt. 36, 165179 (1989).

6. R. Arizaga, M. Trivi, and H. J. Rabal, "Speckle time evolution characterization by the co-occurrence matrix analysis," $\mathrm{Opt}$. Laser Technol. 31, 163-169 (1999).

7. H. Fujii, K. Nohira, Y. Yamamoto, H. Ikawa, and T. Ohura,
"Evaluation of blood flow by laser speckle image sensing. Part 1," Appl. Opt. 26, 5321-5325 (1987).

8. R. Arizaga, N. L. Cap, H. J. Rabal, and M. Trivi, "Display of local activity using dynamic speckle patterns," Opt. Eng. 41, 287-294 (2002)

9. G. H. Sendra, H. J. Rabal, R. Arizaga, and M. Trivi, "Biospeckle images decomposition in temporary spectral bands," Opt. Lett. 30, 1641-1643 (2005).

10. A. Federico, G. H. Kaufmann, G. E. Galizzi, H. Rabal, M. Trivi, and R. Arizaga, "Simulation of dynamic speckle sequences and its application to the analysis of transient processes," Opt. Commun. 260, 493-499 (2006).

11. T. F. Begemann, "Optical measurement of deformation fields and surface processes with digital speckle correlation," Ph.D. dissertation (University of Oldenburg, 2002).

12. T. Fricke-Begemann, G. Gülker, K. D. Hinsch, and K. Wolff, "Corrosion monitoring with speckle correlation," Appl. Opt. 38, 5948-5955 (1999).

13. M. Pajuelo, G. Baldwin, R. Arizaga, N. Cap, H. Rabal, and M. Trivi, "Bio-speckle assessment of bruising in fruits," Opt. Lasers Eng. 40, 13-24 (2003).

14. I. Passoni, H. Rabal, and C. Arizmendi, "Characterizing dynamic speckle time series with the Hurst coefficient concept," Fractals 12, 319-329 (2004).

15. I. Passoni, A. Dai Pra, H. Rabal, M. Trivi, and R. Arizaga, "Dynamic speckle processing using wavelets based entropy," Opt. Commun. 246, 219-228 (2005).

16. J. D. Briers, "The statistics of fluctuating speckle patterns produced by a mixture of moving and stationary scatterers," Opt. Quantum Electron. 10, 364-366 (1978). 